\title{
Rationale, Design for the ASSET Study: A Prospective Randomized Study Comparing Empagliflozin's Effect to Sitagliptin on Cardiac Fat Accumulation/Function in Patients with Type 2 Diabetes
}

\author{
Fumika Shigiyama - Shigenori Hiruma - Shinji Hisatake • \\ Nobuyuki Shiraga - Takanori Ikeda · Takahisa Hirose • \\ Naoki Kumashiro (iD
}

Received: April 13, 2019 / Published online: June 6, 2019

(C) The Author(s) 2019

\begin{abstract}
Introduction: Ectopic fat accumulation has been found to play a pathophysiological role in insulin resistance, type 2 diabetes (T2DM), and coronary artery diseases. Findings from a number of previous studies suggest that sodium glucose cotransporter 2 (SGLT2) inhibitors reduce lipid accumulation, including myocardial and pericardial fat, while dipeptidyl peptidase 4 (DPP4) inhibitors suppress ectopic lipid accumulation and improve cardiac function.
\end{abstract}

Fumika Shigiyama and Shigenori Hiruma contributed equally to this article.

Enhanced Digital Features To view enhanced digital features for this article go to https://doi.org/10.6084/ m9.figshare.8166668.

F. Shigiyama $\cdot$ S. Hiruma $\cdot$ T. Hirose $\cdot$ N. Kumashiro $(\bowtie)$

Division of Diabetes, Metabolism and

Endocrinology, Department of Medicine, Toho

University Graduate School of Medicine, 6-11-1

Omori-Nishi, Ota-ku, Tokyo, Japan

e-mail: naoki.kumashiro@med.toho-u.ac.jp

S. Hisatake $\cdot$ T. Ikeda

Division of Cardiovascular, Department of

Medicine, Toho University Graduate School of

Medicine, 6-11-1 Omori-Nishi, Ota-ku, Tokyo, Japan

\section{N. Shiraga}

Division of Radiology, Department of Medicine,

Toho University Graduate School of Medicine, 6-11-

1 Omori-Nishi, Ota-ku, Tokyo, Japan
However, a clinical study that precisely explains and compares the efficacy of SGLT2 inhibitors and DPP4 inhibitors on cardiac fat accumulation has not been performed. Moreover, the association between cardiac fat accumulation and cardiac function or metabolic changes, such as tissue-specific insulin resistance, remains unclear. It is our intention to conduct the first study to assess the effects of empagliflozin compared to sitagliptin in reducing ectopic fat accumulation, specifically pericardial fat, and its association with improvement in cardiac function and tissue-specific insulin sensitivity.

Methods: We have designed a prospective, randomized open-label, and blinded-endpoint study with the intention to enroll 44 Japanese patients with T2DM. The patients are to be divided them into two groups, an empagliflozin group and an sitagliptin group, with the former to be supplemented with empagliflozin $10 \mathrm{mg}$ and the latter to be supplemented with sitagliptin $100 \mathrm{mg}$, both groups for 12 weeks. The primary endpoint of the study is the change in the amount of pericardial fat. The secondary endpoints are the changes in the amount of intracellular fat in the myocardium, cardiac function, tissue-specific insulin sensitivity, fatty acid metabolism in myocardial tissue, assessed by parameters of iodine-123- $\beta$-methyl-iodophenyl pentadecanoic acid myocardial scintigraphy, blood and urine biomarkers, and lifestyle evaluation. 
Planned Outcomes: The results of this study will be available in 2020. The aim of this study is to provide an effective treatment strategy for patients with T2DM by considering cardiac fat accumulation, cardiac function, and insulin resistance.

Funding: Boehringer Ingelheim \& Eli Lilly and Company Diabetes Alliance.

Trial Registration: University Hospital Medical Information Network Clinical Trial Registry: UMIN000026340.

Keywords: Cardiac function; DPP4 inhibitor; Pericardial fat; SGLT2 inhibitor; Type 2 diabetes

\section{INTRODUCTION}

Ectopic fat accumulation plays a central role in the induction of insulin resistance in type 2 diabetes (T2DM) [1-3], and the association between cardiovascular diseases (CVD) and this form of accumulation has recently been reported [4-6]. Pericardial and myocardial fat accumulation are well-known to result in the excessive release of proinflammatory cytokine and free fatty acid, which causes myocardial intracellular lipotoxicity, possibly inducing myocardial fibrosis and cardiac dysfunction [7-9]. Reducing pericardial fat content and myocardial intracellular lipid content may therefore ameliorate cardiac dysfunction and lower the risk of cardiovascular events.

Some previous studies have demonstrated the effect of anti-diabetic oral agents in improving peripheral lipid accumulation via epicardial fat [10-14]. The effects of dipeptidyl peptidase 4 (DPP4) inhibitors on improving peripheral lipid accumulation have been reported $[15,16]$. Sitagliptin is one of the DPP4 inhibitors widely used to treat T2DM. In addition, it exerts a favorable effect on the reduction of hepatic steatosis [17] and epicardial fat [11] in humans. Although it has been suggested that DPP4 inhibitors ameliorate cardiac function [18], some randomized clinical trials (RCTs) have shown their non-inferiority on cardiovascular events in patients with T2DM compared to the placebo controls [19-21]. In contrast, sodium glucose cotransporter 2 (SGLT2) inhibitors, especially empagliflozin, canagliflozin, and dapagliflozin, have gained increased attention since a RCT addressed their effects on cardiovascular event suppression among patients with T2DM $[22,23]$. SGLT2 inhibitors can prevent the elevation of blood glucose levels by suppressing the reuptake of sodium and glucose from primitive urine [24]. A number of previous studies revealed that SGLT2 inhibitors have multiple metabolic effects, such as decreasing blood pressure [25], reducing triglyceride [26], body weight [27], and fat accumulation in the liver [28], and improving insulin resistance [29]. Furthermore, SGLT2 inhibitors were recently reported to reduce epicardial fat accumulation [12-14] and are suggested to act directly on the heart and neurohumoral factors to suppress cardiovascular events rather than arteriosclerosis [12]. It is also possible that SGLT2 inhibitors also change fuel utilization toward lipids from glucose [30]. Thus, both DPP-4 inhibitors and SGLT2 inhibitors have favorable metabolic effects and may improve cardiac lipid accumulation and function.

To date, however, there is no clinical study that precisely explains and compares the efficacy of SGLT2 inhibitors and DPP4 inhibitors on cardiac lipid accumulation. Moreover, evidence of their relation to metabolic changes and cardiac function, such as tissue-specific insulin resistance, remains unknown. Therefore, our intention is to clinically evaluate the cardioprotective effects of SGLT2 inhibitors, using empagliflozin, on the amount of pericardial fat in comparison to the most commonly used DPP4 inhibitor, sitagliptin. A second aim is to perform a comprehensive assessment of cardiac function, myocardial fatty acid metabolism, tissue-specific insulin sensitivity using the hyperinsulinemic-euglycemic clamp test, lipid accumulation in liver and muscle, whole body composition, and blood and urine biomarkers, as well as a lifestyle evaluation.

To our knowledge, this is the first study to assess the effects of empagliflozin on reducing ectopic fat accumulation, specifically pericardial fat, and improving cardiac function and tissuespecific insulin sensitivity compared to sitagliptin. In this study, we also plan to evaluate 
the effect of SGLT2 inhibitors and DPP4 inhibitors from various metabolic viewpoints. The results of this study will provide new strategies against peripheral lipid accumulation and risk management of CVD with T2DM.

\section{METHODS}

\section{Study Design}

The ASSET study is an ongoing, prospective, randomized open-label, blinded-endpoint study, registered with the University Hospital Medical Information Network Clinical Trial Registry (UMIN000026340), a non-profit organization in Japan that meets the requirements of the International Committee of Medical Journal Editors (ICMJE). This study is approved by the certified clinical research review board of Toho University (CRB3180016) and is being conducted according to the Declaration of Helsinki and its later amendments or comparable ethical standards, as well as current legal regulations in Japan. To avoid bias regarding the collected data, the processes of enrollment, randomization, data collection, and management have been conducted by a third-party. Informed consent is obtained from all individual participants included in the study.

\section{Study Population}

In this study, the target number of patients required for registration was 44 Japanese patients with T2DM. Recruitment for the study began in April 2017 and ended in March 2019 at the Toho University Omori Medical center. The inclusion criteria were: (1) T2DM patients with proper diet and exercise therapy alone or prescribed $\alpha$-glucosidase inhibitors, sulfonylureas, glinides, or combinations of these agents; (2) patients with glycated hemoglobin (HbA1c) levels of $\geq 6.0 \%$ but $\leq 10.0 \%$; (3) patients between 20 and 74 years of age; (4) patients with body mass index (BMI) of $\geq 22 \mathrm{~kg} / \mathrm{m}^{2}$; and (5) patients who provide written informed consent. The exclusion criteria were: (1) type 1 diabetes mellitus or secondary diabetes mellitus patients; (2) patients with BMI of $<22 \mathrm{~kg} / \mathrm{m}^{2}$; (3) patients with renal dysfunction (estimated glomerular filtration rate of $<45 \mathrm{~mL} / \mathrm{min} /$ $1.73 \mathrm{~m}^{2}$ ); (4) patients with medical history of cerebral infarction or stroke within 12 weeks before giving consent for enrollment; (5) patients with a past medical history of myocardial infarction or angina pectoris, or with a present medical history of atrial fibrillation; (6) patients with left ventricular (LV) ejection fraction of $<30 \%$; (7) patients with infection; (8) patients with any untreated cancer; (9) patients with collagen diseases, with the exception of those whose disease progresssion is well controlled with prednisolone at a dose of $\leq$ $5 \mathrm{mg} /$ day; (10) patients with hepatic cirrhosis; (11) patients with liver failure that is either virus-, autoimmune-, or drug-induced; (12) alcoholics; (13) pregnant or breastfeeding patients, or those planning to become pregnant during this study; (14) patients allergic to empagliflozin or sitagliptin; and (15) patients with anemia (hemoglobin $<12 \mathrm{~g} / \mathrm{dL}$ ).

\section{Randomization and Study Intervention}

The schedule for enrollment, randomization, and follow-up that are being used in this study is shown in Fig. 1. After consent had been obtained and the patients enrolled, clinical and biochemical analysis, cardiac magnetic resonance imaging (MRI), proton magnetic resonance spectroscopy $\left({ }^{1} \mathrm{H}-\mathrm{MRS}\right)$ analysis, iodine123- $\beta$-methyl-iodophenyl pentadecanoic acid myocardial scintigraphy ( ${ }^{123}$ I-BMIPP scintigraphy), echocardiography, indirect calorimetry, and hyperinsulinemic-euglycemic clamp would be performed for each subject within 10 weeks. Eligible subjects would be randomly and equally assigned to the empagliflozin group (prescribed empagliflozin $10 \mathrm{mg} /$ day) or sitagliptin group (prescribed sitagliptin $50 \mathrm{mg} /$ day as primary dose). Randomization would be performed by a computer-based dynamic allocation method using the presence of sulfonylureas administration and intrahepatic lipid content as assessed by MRS at the baseline checkup. After treatment initiation, a medical check for vital signs, urinalysis, and blood tests would be performed to 


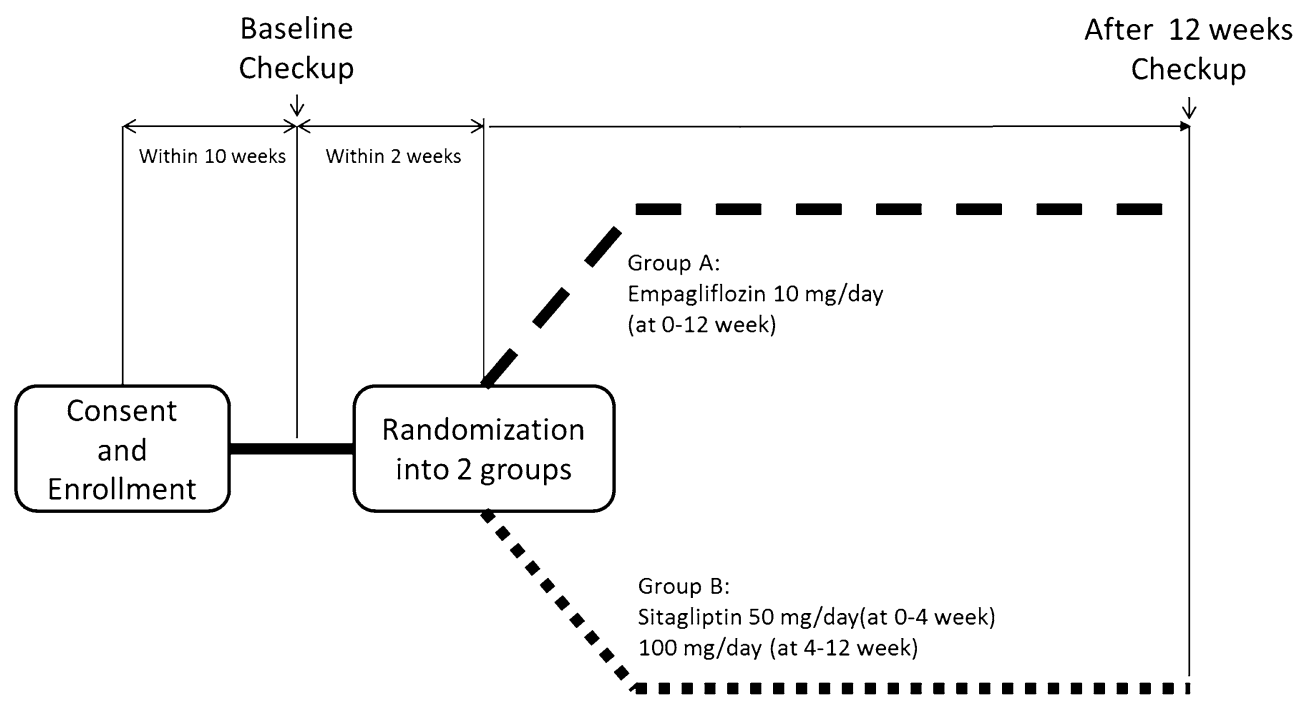

Fig. 1 After consent and enrollment, clinical and biochemical analysis, cardiac magnetic resonance imaging, proton magnetic resonance spectroscopy, iodine-123- $\beta$ methyl-iodophenyl pentadecanoic acid myocardial scintigraphy, echocardiography, indirect calorimetry, and hyperinsulinemic euglycemic clamp would be performed on the subject within 10 weeks. The participant would then be randomly assigned to the empagliflozin group or sitagliptin

screen for the occurrence of adverse events at 4 weeks. Concurrently, the dosage of sitagliptin would be increased to $100 \mathrm{mg} /$ day for the sitagliptin group. After the 12-week treatment period, the same tests and checks carried out at baseline would be performed. All measures of parameters to be evaluated would be collected after overnight fasting.

\section{Study Endpoints}

The primary endpoint of this study is the change in the amount of pericardial fat, which is the sum of epicardial and paracardial fat, as evaluated by MRI. The secondary endpoints are the following indices for changes from baseline to 12 weeks: (1) intracellular fat in the myocardium, muscle, and liver; (2) cardiac function estimated by echocardiography and cine-MRI; (3) extracellular fat in the muscle assessed by ${ }^{1} \mathrm{H}$-MRS; (4) insulin sensitivity in the liver, muscle and adipose tissue assessed by the hyperinsulinemic-euglycemic clamp method; (5) indicator of cardiac fatty acid metabolism group. In the sitagliptin group, the dosage of sitagliptin would be increased by $100 \mathrm{mg} /$ day at 4 weeks. After the 12-week medication period, the same assessments as those carried out at baseline would be performed. All assessments were performed after an overnight fasting

assessed by ${ }^{123}$ I-BMIPP scintigraphy; (6) indicators of energy metabolism assessed by indirect calorimetry (respiratory quotient, energy expenditure, oxygen consumption, carbon dioxide emissions); (7) plasma biomarkers (Cpeptide, plasma insulin, renin activity, aldosterone, brain natriuretic peptide, heart type fatty acid-binding protein, high-sensitivity C-reactive protein [CRP], adiponectin, leptin, and metabolome assay); (8) urine biomarkers (urine general test, microalbumin, glucose, ketone, sodium $[\mathrm{Na}]$, potassium $[\mathrm{K}]$, chlorine $[\mathrm{Cl}]$, creatinine [Cre], and 8-hydroxy-2'-deoxyguanosine); (9) body weight, blood pressure, BMI, body composition (total body water, bone mass, muscle mass, fat content, body fat percentage, and basal metabolic rate); (10) lifestyle assessment, including precise dietary intake using the brief-type self-administered diet history questionnaire (BDHQ) [31], (11) medication compliance; and (12) incidence of adverse event. 


\section{Cardiac Magnetic Resonance Imaging}

Assessment of cardiac fat accumulation and cardiac function would be performed by cineMRI. The cardiac imaging protocol was used for MRI with reference to previous reports [32, 33]. In brief, cardiac MRI with a $1.5 \mathrm{~T}$ whole-body MR scanner (MAGNETOM AvantoSQ1.5T B-19; Siemens, Erlangen, Germany) would be used to evaluate cardiac fat accumulation and cardiac function in the breath-holding position. The cine series would be obtained in the fourchamber, two-chamber, and LV short axis views using a steady-state free precession sequence. A number of cine images would be used to cover the LV from the base to apex. Scanning would be performed with typical imaging parameters: repetition time $68.4 \mathrm{~ms}$; echo time $1.48 \mathrm{~ms}$; flip angle $80^{\circ}$; matrix $134 \times 192$; field of view $360 \times 360 \mathrm{~mm}$; slice thickness $10 \mathrm{~mm}$; gap, $0 \mathrm{~mm}$; and calculated phases 25 . Estimation of epicardial fat and paracardial fat would be performed by a non-participating doctor using a dedicated software (SYNAPSE VINCENT; Fujifilm Corp., Tokyo, Japan). The high signal range between the myocardium and pericardium would be considered to be epicardial fat; similarly, the high signal range outside the pericardium would be considered to be paracardial fat. The data for the pericardium, which is the primary endpoint of this study, would be obtained from the sum of epicardial fat and paracardial fat.

Cardiac function data of the LV would also be evaluated using MRI by experienced technicians blinded to the clinical background of subjects with a dedicated software (Argus; Siemens, Erlangen, Germany). LV ejection fraction, cardiac mass, end-diastolic volume, endsystolic volume, stroke volume, peak ejection rate, peak filling rate, time to peak filling rate, and heart rate would be measured.

\section{Echocardiography}

Echocardiography would be performed by experienced technicians with $3.5-$ and $2.5-\mathrm{MHz}$ transducers for two-dimensional, M-mode, and continuous-wave Doppler measurements. LV end-systolic diameter (LVDs), LV end-diastolic diameter (LVDd), cardiac mass, cardiac volumes, LV ejection fraction, and the ratio between early mitral inflow velocity and mitral annular early diastolic velocity $\left(E / e^{\prime}\right)$ would be evaluated. The percentage fractional shortening of the ventricle (\%FS) would be calculated using the formula: $\% \mathrm{FS}=($ LVDd - LVDs $) \times 100 /$ LVDd [34].

\section{Proton MRS Analysis}

A ${ }^{1} \mathrm{H}$-MRS would be performed to measure cardiac fat accumulation using a $1.5 \mathrm{~T}$ whole-body MR scanner (MAGNETOM AvantoSQ 1.5 T B-19; Siemens) run by specialists using dedicated software (Argus; Siemens). The method used to measure cardiac fat accumulation with ${ }^{1} \mathrm{H}$-MRS has been previously validated [35-37]. The volume of interest $\left(\mathrm{VOI}=10 \times 10 \times 20 \mathrm{~mm}^{3}\right)$ would be manually placed on the ventricular septum of the cine images of the heart and adjusted to fit the ventricular septum of the left ventricle. The spectra of lipid and water would be acquired by point-resolved spectroscopy sequences (repetition time/echo time ms $4000 / 30$ ). The myocardial signal would be quantified as triglyceride signal intensity at $1.4 \mathrm{ppm}$ from the spectra with water suppression, and water signals would be quantified at $4.7 \mathrm{ppm}$ from the spectra without water suppression.

Similarly, VOI $\left(20 \times 20 \times 20 \mathrm{~mm}^{3}\right)$ would be set on the liver and tibialis anterior muscle on the acquired MR data, avoiding liver edges, visible blood vessels, or bile ducts. The MR spectral raw data would then be processed to calculate intrahepatic lipid content, intramyocellular lipid content, and extramyocellular lipid content using LC model software (version 6.3-1J; Stephen Provencher, Oakville, ON, Canada). Each lipid would be estimated by an indicator as a ratio of lipid to water.

\section{Hyperinsulinemic-Euglycemic Clamp}

Tissue-specific insulin sensitivity would be assessed by the hyperinsulinemic-euglycemic clamp method using an artificial pancreas 
system (STG55; Nikkiso, Tokyo, Japan) as previously described [38] after overnight fasting. Subjects would lie on a bed, and three intravenous catheters would be placed on their forearms. Each catheter would be used for tracer and insulin infusion via connection to the artificial pancreas and for blood sampling, respectively. The arm for blood sampling, which would be connected to the artificial pancreas, would be kept warm by an electric blanket to derive the parameters of venous blood under the arterial blood condition. At the start of the 3-h basal infusion period, 6,6$\left[{ }^{2} \mathrm{H}_{2}\right]$ glucose $(99 \%$ atom percent enrichment [APE]; Cambridge Isotope Laboratories Inc., Andover, MA, USA) would be infused at a priming dose $(200 \times$ fasting plasma glucose $[\mathrm{mg} / \mathrm{dL}] / 100 \mathrm{mg} / \mathrm{m}^{2}$ body surface area) and continued at $2 \mathrm{mg} / \mathrm{m}^{2}$ body surface area/min.

After this period, the hyperinsulinemic-euglycemic clamp test would be initiated. Insulin (U-100 Humulin R; Eli Lilly, Indianapolis, IN, USA) would be infused at 160 and $80 \mathrm{mU} / \mathrm{m}^{2} / \mathrm{min}$, each infusion rate for $5 \mathrm{~min}$, as a priming dose. After $10 \mathrm{~min}$, the insulin infusion dise would be changed to $40 \mathrm{mU} / \mathrm{m}^{2} / \mathrm{min}$ for the continuous dose [38-40].

A variable infusion of $20 \%$ glucose containing $2.5 \% \quad 6,6-\left[{ }^{2} \mathrm{H}_{2}\right]$ glucose would be administered via the artificial pancreas to maintain the plasma glucose concentration at approximately $95 \mathrm{mg} / \mathrm{dL}$ [41]. During the clamp test, blood samples would be collected from the catheter line at $-180,-30,-15,0,15,30,60,120,160$, 170 , and $180 \mathrm{~min} .{ }^{2} \mathrm{H}$ APE in plasma glucose would be measured by high-performance liquid chromatography using a LTQ Orbitrap XL mass spectrometer (Thermo Scientific, Fremont, CA, USA). Basal and clamped endogenous glucose production and the clamped glucose disposal rate would be evaluated using a steady-state equation as described previously [38, 42]. Clamped percentage suppression of serum free fatty acid by insulin, which represents the insulin sensitivity of adipose tissue, would be calculated as: (serum free fatty acid concentration at baseline - mean free fatty acid concentration at time $160-180 \mathrm{~min}$ ) divided by free fatty acid concentration at baseline $\times 100$.

\section{${ }^{123}$ I-BMIPP Scintigraphy}

After an intravenous injection of $111 \mathrm{MBq}$ of ${ }^{123} \mathrm{I}$-BMIPP radiotracer (111 MBq/1.5 mg; Nihon Medi-Physics Co. Ltd., Tokyo, Japan), early images would be captured at 20 min and delay images would be taken at $3 \mathrm{~h}$, both by a dualheaded single-photon emission computed tomography (SPECT) gamma camera (Infinia H3000WT; GE Medical System, Tel Aviv, Israel). SPECT images would be acquired in a step shooting mode using two detectors $\left(180^{\circ}\right.$ rotation) and at a matrix size of $64 \times 64$. A series of contiguous transaxial images with a thickness of $5.89 \mathrm{~mm}$ would be reconstructed using the Butterworth filtered back projection algorithm (order 10; cut-off 0.40 cycles $/ \mathrm{cm}$ ) without attenuation or correction.

Regional tracer uptake would be scored, semiquantatively, from 0 (normal) to 4 (severe defect) for 17 segments of the left ventricle, and the sum of the defect scores for all segments would be calculated to derive the summed rest score (SRS) [43].

The washout rate of the heart between $20 \mathrm{~min}$ and $3 \mathrm{~h}$ after intravenous injection of the ${ }^{123}$ I-BMIPP would be calculated by the following formula: (count at $20 \mathrm{~min}$ - count at $3 \mathrm{~h}$ )/count at $20 \mathrm{~min} \times 100$.

The count per pixel data would be measured for both the heart and the mediastinum, and the ratio of heart $(H)$-to-mediastinal $(M)$ uptake $(H / M)$ at 20 min (early phase) and $3 \mathrm{~h}$ (delay phase) after the injection of ${ }^{123}$ I-BMIPP would be calculated [44].

\section{Indirect Calorimetry}

Energy expenditure, oxygen consumption $\left(\mathrm{VO}_{2}\right)$, carbon dioxide production $\left(\mathrm{VCO}_{2}\right)$, and respiratory quotient (RQ) would be measured by indirect calorimetry using a gas analysis device (Metalyzer 3B; Cortex, Leipzig, Germany). Subjects, in fasting condition and lying on a bed, would wear a dedicated mask that was connected to the device. They would then be asked to breathe normally for $30 \mathrm{~min}$ in the quiet environment of the isolated room. To assess the indirect calorimetry data, a 5-min interval of 
data of the entire 30-min dataset would be selected as the steady-state condition. Steadystate indicates that the respiratory quotient remains $<10 \%$, even when it fluctuates. The RQ would be calculated by the following formula; $\mathrm{RQ}=\mathrm{VCO}_{2}(\mathrm{~L} / \mathrm{min}) / \mathrm{VO}_{2}(\mathrm{~L} / \mathrm{min})$.

\section{Clinical and Biochemical Analysis}

Blood and urine samples collected at Toho University Hospital would either be submitted to the central laboratory of the hospital or to a private laboratory (SRL laboratory, Tokyo, Japan) where they would be analyzed by the following methods: a chemiluminescent immunoassay for C-peptide, brain natriuretic peptide, adiponectin, ferritin, and plasma insulin; a direct method for high-density lipoprotein cholesterol and lowdensity lipoprotein cholesterol; double antibody radioimmunoassay for leptin and the $7 \mathrm{~S}$ domain of type IV collagen; an electrode method for $\mathrm{Na}$, $\mathrm{K}$, and $\mathrm{Cl}$; an enzymatic method for triglycerides, creatinine, uric acid, amylase, ketone body fraction, and free fatty acid; high-performance liquid chromatography for urinary 8-hydroxy-2'-deoxyguanosine; The Japan Society for Clinical Chemistry transferable method for aspartate aminotransferase, alanine aminotransferase, and $\gamma$-glutamyl transpeptidase; the latex agglutination method for heart-type fatty acid-binding protein and HbA1c; nephelometry for plasma albumin; radioimmunoassay for plasma renin activity and aldosterone; a sandwich enzymelinked immunosorbent assay for plasma glucagon; a test paper method for urine general test; turbidimetric immunoassay for apolipoproteins and urinary microalbumin; ultraviolet absorption spectrophotometry for total cholesterol, urea nitrogen, and glucose; latex agglutination turbidimetric immunoassay for hyaluronic acid; electro-chemiluminescence immunoassay for cortisol; and chemiluminescent immunoassay for type III procollagen- $\mathrm{N}$-peptide.

\section{Assessment for Body Composition}

Body composition would be measured by an experienced examiner using a whole body tetrapolar bioimpedance analyzer (InBody230;
Biospace, Seoul, South Korea). The subject would stand (bare foot) on the analyzer and grasp its handles. Investigators would record the patient's age, sex, and height. Five body segments (both arms, both legs, and trunk) would then be analyzed using different frequencies (20 and $100 \mathrm{kHz}$ ). The analyzer calculates the types of body compositional parameters, such as fatfree-mass, fat mass, and percentage body fat.

\section{Safety and Evaluation of Adverse Events}

During this study, the investigators would constantly monitor any adverse events (AEs) through regular medical checkups. All related AEs, not only side effects to the drug but also abnormal values from the clinical tests, would be reported and documented.

\section{Sample Size Estimation}

To our knowledge, this study is the first attempt to compare and identify the better suited medication, empagliflozin (a SGLT2 inhibitor) or sitagliptin (a DPP4 inhibitor) for improving cardiac fat accumulation or function. Therefore, a statistical basis for the chosen target number of participants does not exist. As the reduction of lipid accumulation in the liver is associated with the improvement in cardiac insulin resistance [45], we referred to previous studies with seven Japanese subjects [17] and 50 US subjects [46] that addressed the reduction in intrahepatic lipid content by sitagliptin administration. Thus, we estimated that 44 would be a sufficient and feasible number of subjects for inclusion.

\section{Statistical Analysis}

Analysis for the primary and secondary endpoints would be primarily performed on the full analysis set (FAS). FAS includes all research subjects enrolled in this study and assigned to a study treatment. However, subjects without data for the primary endpoint or subjects with a significant study protocol violation would be excluded. Safety analysis with AEs would be performed on the treated set. Summary statistics would be calculated for continuous 
variables. For comparisons between categorical variables of the two groups, the chi-square test or Fisher's exact test would be used for nominal variables, and the two-sample $t$ test or Wilcoxon ranked-sum test would be used for continuous variables.

\section{Human Rights and Ethical Principles of Study Subjects}

All investigators involved in this study are in compliance with the "World Medical Association Declaration of Helsinki" (2013 revision), with the "Ethical Guidelines for Medical and Health Research Involving Human Subjects" (December 22, 2014, Ministry of Education, Culture, Sports, Science and Technology/Ministry of Health, Labor and Welfare), and with other laws and regulations. All statistical analyses will be performed independently by the administrative office of the ASSET study using SAS software version 9.4 (SAS Institute, Cary, NC, USA).

\section{DISCUSSION}

To our knowledge, this will be the first study to compare the SGLT2 inhibitor, empagliflozin, to the DPP4 inhibitor, sitagliptin, for the amelioration of cardiac fat accumulation, cardiac function, and tissue-specific insulin sensitivity in Japanese patients with T2DM.

For the current study, pericardial fat (epicardial fat and paracardial fat) is set as the primary endpoint. Many methods to determine the amount of pericardial fat have been previously reported [32, 47-49]. Of these methods, cine-MRI can concurrently measure pericardial fat and cardiac function. Graner et al. [32] reported that the area measured as epicardial and paracardial fat in a single 4-chamber image assessed by cine-MRI showed a good correlation with the volumes measured by the conventional Simpson method which covers both right and left ventricles in a stack of short-axis image slices. Therefore, we considered that the fourchamber MR image at the end-diastolic state is accurate in estimating the amount of epicardial and paracardial fat and selected this method for use in this study.

Both pericardial fat and myocardial triglyceride content are considered to be risk factors for cardiac dysfunction [50]. To estimate the risk of CVD or heart failure before their onset, it is important to evaluate cardiac function. Luuk et al. [51] showed that myocardial triglyceride content was increased in patients with T2DM and proposed that this increase impaired LV diastolic function. In support of this proposal, Mikko et al. [52] reported that myocardial triglyceride content correlates with pericardial fat. Therefore, myocardial triglyceride content was set as one of the secondary endpoints of this study. It can be evaluated by ${ }^{1} \mathrm{H}-\mathrm{MRS}$, a validated, non-invasive, and useful method to evaluate the content of this particular parameter [35-37].

The EMPA-REG outcomes trial revealed that empagliflozin prevents patients with diabetes and CVD from not only 3-point major adverse $\mathrm{CV}$ events (MACE), but also hospitalization due to heart failure [22]. Surprisingly, in this outcomes trial hospitalization due to heart failure was reduced by treatment with empagliflozin compared to placebo after only 12 weeks of intake. Although it has been considered that diabetes causes CVD through arteriosclerosis progression, the suppression of hospitalization due to heart failure is unlikely to be ascribed to the suppression of arteriosclerosis in only 12 weeks. Therefore, empagliflozin might have other cardiac benefits in addition to protecting against arteriosclerosis. In this study, we hypothesized that empagliflozin is superior to DPP4 inhibitors in improving cardiac function via reducing pericardial lipid accumulation. Some researchers have already reported that SGLT2 inhibitors reduce epicardial fat accumulation and improve cardiac function [12-14]. Habibi et al. [53] also proved that empagliflozin improves cardiac diastolic function in a rodent model of diabetes; however, but this was a single-arm study and it is therefore still unknown whether SGLT2 inhibitors are superior to other glucose-lowering agents in reducing cardiac lipid accumulation. In Japan, however, sitagliptin, a DPP4 inhibitor, is to date the most widely prescribed anti-diabetic oral agent and 
might have the effect of reducing ectopic fat, including epicardial fat $[11,17,46]$. Based on this information, we selected sitagliptin for use as the comparator drug in this study. As other SGLT-2 inhibitors have been found to reduce pericardial fat after only 12 weeks of medication intake $[12,14]$, the study period was set as 12 weeks for this study.

Both empagliflozin and sitagliptin are known to improve various metabolic parameters as well as possess glucose-lowering effects. Previous studies have found that the administration of SGLT2 inhibitors resulted in lowered blood pressure [54] and reduced uric acid and oxidative stress [55]. In contrast, DPP4 inhibitors have been found to reduce inflammatory markers such as CRP [56, 57] and apolipoprotein B [58], and increased adiponectin [59]. Thus, in this study, our aim is to evaluate various parameters, such as tissue-specific insulin sensitivity, cardiac function, cardiac fatty acid metabolism, energy expenditure, among others. To evaluate insulin sensitivity in the liver, muscle, and adipose tissue, the hyper-insulinemic-euglycemic clamp is scheduled for use in this study. As SGLT2 inhibitors reduce body weight and lipid accumulation, insulin resistance may improve. In fact, it has been reported that SGLT2 inhibitors improve insulin resistance in humans [60] and rodents [61]. Additionally, the parameters of ${ }^{123}$ I-BMIPP scintigraphy, which can estimate the cardiac fatty acid metabolism, will be measured in this study. Furthermore, we will use the indirect calorimetry method to determine the $R Q$, which indicates the ratio of carbohydrate and lipid as energy substrates. These results should provide a further understanding of the effect of SGLT2 and DPP4 inhibitors to reveal the better therapeutic choice.

While we intend to precisely compare the effects of empagliflozin and sitagliptin on cardiac fat accumulation and various metabolic parameters, this study has several limitations. First, it is an open-label study, and subjects can be informed of their medication details, which may affect the results. Second, all patients are Japanese and the study's duration is short. Third, to estimate insulin sensitivity in cardiac muscle, a more suitable method would have been the cardiac proton emission tomography (PET) method with a stable isotope [62]; however, we cannot perform this method at the study site due to our faculty's lack of the appropriate instrumentation and expertise. Finally, all subjects do not have CVD; thus, this study will reveal information limited to primary prevention and will leave the effects on secondary prevention unclear.

\section{CONCLUSION}

Through the ASSET study, we intend to elucidate the effects of empagliflozin on pericardial fat, myocardial fat, cardiac function, and tissuespecific insulin sensitivity compared to sitagliptin. No prior studies have assessed and compared this many cardiometabolic parameters in a comprehensive manner. In addition, this study will reveal the potential benefits of empagliflozin, and the results will enable us to propose an effective treatment strategy against cardiac lipid accumulation and function in T2DM. The results of this study will be available in 2020.

\section{ACKNOWLEDGEMENTS}

We thank all the participants of this study.

Funding. This study was funded by the Boehringer Ingelheim \& Eli Lilly and Company Diabetes Alliance. The journal's article processing charges were also funded by them. Both companies had no role in the study design, data collection and analysis, decision to publish, or preparation of the manuscript. All authors had full access to all of the data in this study and take complete responsibility for the integrity of the data and accuracy of the data analysis.

Medical Writing and/or Editorial Assistance. We also sincerely appreciate Hiroyuki Igarashi, Hideaki Suzuki and Sunao Mizunuma for their excellent technical support. We would like to thank Editage (http://www.editage.jp) for English language editing. 
Authorship. All named authors meet the International Committee of Medical Journal Editors (ICMJE) criteria for authorship for this article, take responsibility for the integrity of the work as a whole, and have given their approval for this version to be published.

Authors Contribution. FS, SH, NK conducted the study. FS, SH, and NK wrote and edited the manuscript. TH contributed to the discussion. SH and TI managed the cardiac examinations, such as echocardiography and iodine-123- $\beta$-methyl-iodophenyl pentadecanoic acid myocardial scintigraphy. NS managed image examinations, such as cardiac magnetic resonance imaging and proton magnetic resonance spectroscopy. NK is the guarantor of this work and, as such, had full access to all study data and takes responsibility for the integrity of the data and the accuracy of data analysis.

Disclosures. Naoki Kumashiro has received research funds from Boehringer Ingelheim Pharmaceuticals, Inc., and lecture fees from Novo Nordisk Inc., Takeda Pharmaceutical Company Limited, Ono Pharmaceutical Co., Ltd., and Sanofi-Aventis Deutschland GmbH. Takahisa Hirose has received research funds from AstraZeneca, Boehringer Ingelheim Pharmaceuticals, Inc., Ono Pharmaceutical Co., Ltd., Novo Nordisk Inc., Sanofi-Aventis Deutschland GmbH, Daiichi-Sankyo Co., Ltd., Eli Lilly Japan K.K., Takeda Pharmaceutical Company Limited, Mitsubishi Tanabe Pharma Corporation, Dainippon Sumitomo Pharma Co., Ltd., and Kissei Pharmaceutical Co., Ltd., and lecture fees from Sanofi-Aventis Deutschland GmbH, Eli Lilly Japan K.K., Novo Nordisk Inc., Takeda Pharmaceutical Company Limited, Daiichi-Sankyo Co., Ltd., Mitsubishi Tanabe Pharma Corporation, Merck \& Co., Inc., Dainippon Sumitomo Pharma Co., Ltd., Novartis Pharma K.K., Kissei Pharmaceutical Co., Ltd., Boehringer Ingelheim Pharmaceuticals, Inc., Ono Pharmaceutical Co., Ltd., and AstraZeneca. All funding agencies played no role in the study design, data collection and analysis, decision to publish, or preparation of the manuscript. Fumika Shigiyama, Shigenori
Hiruma, Shinji Hisatake, Nobuyuki Shiraga, and Takanori Ikeda have nothing to disclose.

Compliance with Ethics Guidelines. All procedures performed in studies involving human participants are in accordance with the ethical standards of clinical research review board of Toho University (CRB3180016) and with the 1964 Helsinki declaration and its later amendments or comparable ethical standards. Informed consent is obtained from all individual participants included in the study.

Data Availability. Data sharing is not applicable to this article as no datasets were generated or analyzed yet.

Open Access. This article is distributed under the terms of the Creative Commons Attribution-NonCommercial 4.0 International License (http://creativecommons.org/licenses/ by-nc/4.0/), which permits any noncommercial use, distribution, and reproduction in any medium, provided you give appropriate credit to the original author(s) and the source, provide a link to the Creative Commons license, and indicate if changes were made.

\section{REFERENCES}

1. Perry RJ, Samuel VT, Petersen KF, Shulman GI. The role of hepatic lipids in hepatic insulin resistance and type 2 diabetes. Nature. 2014;510:84-91.

2. Fabbrini E, Magkos F, Mohammed BS, et al. Intrahepatic fat, not visceral fat, is linked with metabolic complications of obesity. Proc Natl Acad Sci USA. 2009;106:15430-5.

3. Jacob S, Machann J, Rett K, et al. Association of increased intramyocellular lipid content with insulin resistance in lean nondiabetic offspring of type 2 diabetic subjects. Diabetes. 1999;48:1113-9.

4. Schindhelm RK, Diamant M, Heine RJ. Nonalcoholic fatty liver disease and cardiovascular disease risk. Curr Diab Rep. 2007;7:181-7.

5. Targher G, Arcaro G. Non-alcoholic fatty liver disease and increased risk of cardiovascular disease. Atherosclerosis. 2007;191:235-40. 
6. Targher G, Day CP, Bonora E. Risk of cardiovascular disease in patients with nonalcoholic fatty liver disease. N Engl J Med. 2010;363:1341-50.

7. Hirata $\mathrm{Y}$, Tabata $\mathrm{M}$, Kurobe $\mathrm{H}$, et al. Coronary atherosclerosis is associated with macrophage polarization in epicardial adipose tissue. J Am Coll Cardiol. 2011;58:248-55.

8. Shimabukuro M, Hirata Y, Tabata M, et al. Epicardial adipose tissue volume and adipocytokine imbalance are strongly linked to human coronary atherosclerosis. Arterioscler Thromb Vasc Biol. 2013;33:1077-84.

9. Maurovich-Horvat P, Kallianos K, Engel LC, et al. Relationship of thoracic fat depots with coronary atherosclerosis and circulating inflammatory biomarkers. Obesity (Silver Spring). $2015 ; 23: 1178-84$.

10. Sacks HS, Fain JN, Cheema P, et al. Inflammatory genes in epicardial fat contiguous with coronary atherosclerosis in the metabolic syndrome and type 2 diabetes: changes associated with pioglitazone. Diabetes Care. 2011;34:730-3.

11. Lima-Martinez MM, Paoli M, Rodney M, et al. Effect of sitagliptin on epicardial fat thickness in subjects with type 2 diabetes and obesity: a pilot study. Endocrine. 2016;51:448-55.

12. Bouchi R, Terashima M, Sasahara Y, et al. Luseogliflozin reduces epicardial fat accumulation in patients with type 2 diabetes: a pilot study. Cardiovasc Diabetol. 2017;16:32.

13. Yagi S, Hirata Y, Ise T, et al. Canagliflozin reduces epicardial fat in patients with type 2 diabetes mellitus. Diabetol Metab Syndr. 2017;9:78.

14. Fukuda T, Bouchi R, Terashima M, et al. Ipragliflozin reduces epicardial fat accumulation in nonobese type 2 diabetic patients with visceral obesity: a pilot study. Diabetes Ther. 2017;8:851-61.

15. Shirakawa J, Fujii H, Ohnuma K, et al. Diet-induced adipose tissue inflammation and liver steatosis are prevented by DPP-4 inhibition in diabetic mice. Diabetes. 2011;60:1246-57.

16. Kern M, Kloting N, Niessen HG, et al. Linagliptin improves insulin sensitivity and hepatic steatosis in diet-induced obesity. PLoS One. 2012;7:e38744.

17. Watanabe T, Tamura Y, Kakehi S, et al. Effects of sitagliptin on ectopic fat contents and glucose metabolism in type 2 diabetic patients with fatty liver: a pilot study. J Diabetes Investig. 2015;6:164-72.
18. Yamada H, Tanaka A, Kusunose K, et al. Effect of sitagliptin on the echocardiographic parameters of left ventricular diastolic function in patients with type 2 diabetes: a subgroup analysis of the PROLOGUE study. Cardiovasc Diabetol. 2017;16:63.

19. White WB, Cannon CP, Heller SR, et al. Alogliptin after acute coronary syndrome in patients with type 2 diabetes. N Engl J Med. 2013;369:1327-35.

20. Scirica BM, Bhatt DL, Braunwald E, et al. Saxagliptin and cardiovascular outcomes in patients with type 2 diabetes mellitus. $\mathrm{N}$ Engl J Med. 2013;369:1317-26.

21. Green JB, Bethel MA, Armstrong PW, et al. Effect of sitagliptin on cardiovascular outcomes in type 2 diabetes. N Engl J Med. 2015;373:232-42.

22. Zinman B, Wanner C, Lachin JM, et al. Empagliflozin, cardiovascular outcomes, and mortality in type 2 diabetes. N Engl J Med. 2015;373:2117-28.

23. Neal B, Perkovic V, Mahaffey KW, et al. Canagliflozin and cardiovascular and renal events in type 2 diabetes. N Engl J Med. 2017;377:644-57.

24. Kalra S. Sodium glucose co-transporter-2 (SGLT2) inhibitors: a review of their basic and clinical pharmacology. Diabetes Ther. 2014;5:355-66.

25. Tikkanen I, Narko K, Zeller C, et al. Empagliflozin reduces blood pressure in patients with type 2 diabetes and hypertension. Diabetes Care. 2015;38:420-8.

26. Basu D, Huggins LA, Scerbo D, et al. Mechanism of Increased LDL (low-density lipoprotein) and decreased triglycerides with SGLT2 (sodium-glucose cotransporter 2) inhibition. Arterioscler Thromb Vasc Biol. 2018;38:2207-16.

27. Neeland IJ, McGuire DK, Chilton R, et al. Empagliflozin reduces body weight and indices of adipose distribution in patients with type 2 diabetes mellitus. Diab Vasc Dis Res. 2016;13:119-26.

28. Nakano S, Katsuno K, Isaji M, et al. Remogliflozin etabonate improves fatty liver disease in diet-induced obese male mice. J Clin Exp Hepatol. 2015;5:190-8.

29. Joannides CN, Mangiafico SP, Waters MF, Lamont BJ, Andrikopoulos S. Dapagliflozin improves insulin resistance and glucose intolerance in a novel transgenic rat model of chronic glucose overproduction and glucose toxicity. Diabetes Obes Metab. 2017;19:1135-46.

30. Ferrannini E, Baldi S, Frascerra S, et al. Shift to fatty substrate utilization in response to sodium-glucose cotransporter 2 inhibition in subjects without 
diabetes and patients with type 2 diabetes. Diabetes. 2016;65:1190-5.

31. Kobayashi S, Murakami K, Sasaki S, et al. Comparison of relative validity of food group intakes estimated by comprehensive and brief-type selfadministered diet history questionnaires against 16 d dietary records in Japanese adults. Public Health Nutr. 2011;14:1200-11.

32. Graner M, Siren R, Nyman K, et al. Cardiac steatosis associates with visceral obesity in nondiabetic obese men. J Clin Endocrinol Metab. 2013;98:1189-97.

33. Sicari R, Sironi AM, Petz R, et al. Pericardial rather than epicardial fat is a cardiometabolic risk marker: an MRI vs echo study. J Am Soc Echocardiogr. 2011;24:1156-62.

34. Quinones MA, Pickering E, Alexander JK. Percentage of shortening of the echocardiographic left ventricular dimension. Its use in determining ejection fraction and stroke volume. Chest. 1978;74:59-65.

35. Sai E, Shimada K, Yokoyama T, et al. Association between myocardial triglyceride content and cardiac function in healthy subjects and endurance athletes. PLoS One. 2013;8:e61604.

36. Sai E, Shimada K, Yokoyama T, et al. Evaluation of myocardial triglyceride accumulation assessed on 1H-magnetic resonance spectroscopy in apparently healthy Japanese subjects. Intern Med. 2015;54:367-73.

37. Sai E, Shimada K, Yokoyama T, et al. Myocardial triglyceride content in patients with left ventricular hypertrophy: comparison between hypertensive heart disease and hypertrophic cardiomyopathy. Heart Vessels. 2017;32:166-74.

38. Shigiyama F, Kumashiro N, Furukawa Y, et al. Characteristics of hepatic insulin-sensitive nonalcoholic fatty liver disease. Hepatol Commun. 2017;1:634-47.

39. Vozarova B, Stefan N, Lindsay RS, et al. High alanine aminotransferase is associated with decreased hepatic insulin sensitivity and predicts the development of type 2 diabetes. Diabetes. 2002;51:1889-95.

40. Kelley DE, McKolanis TM, Hegazi RA, Kuller LH, Kalhan SC. Fatty liver in type 2 diabetes mellitus: relation to regional adiposity, fatty acids, and insulin resistance. Am J Physiol Endocrinol Metab. 2003;285:E906-16.

41. Korenblat KM, Fabbrini E, Mohammed BS, Klein S. Liver, muscle, and adipose tissue insulin action is directly related to intrahepatic triglyceride content in obese subjects. Gastroenterology. 2008;134:1369-75.

42. Maggs DG, Buchanan TA, Burant CF, et al. Metabolic effects of troglitazone monotherapy in type 2 diabetes mellitus. A randomized, double-blind, placebo-controlled trial. Ann Intern Med. 1998;128:176-85.

43. Matsuki T, Tamaki N, Nakata T, et al. Prognostic value of fatty acid imaging in patients with angina pectoris without prior myocardial infarction: comparison with stress thallium imaging. Eur J Nucl Med Mol Imaging. 2004;31:1585-91.

44. Biswas SK, Sarai M, Toyama $H$, et al. Role of (1)(2)(3)I-BMIPP and serum B-type natriuretic peptide for the evaluation of patients with heart failure. Singapore Med J. 2012;53:398-402.

45. Lautamaki R, Borra R, Iozzo P, et al. Liver steatosis coexists with myocardial insulin resistance and coronary dysfunction in patients with type 2 diabetes. Am J Physiol Endocrinol Metab. 2006;291:E282-90.

46. Cui J, Philo L, Nguyen P, et al. Sitagliptin vs. placebo for non-alcoholic fatty liver disease: a randomized controlled trial. J Hepatol. 2016;65:369-76.

47. Hirata Y, Yamada H, Kusunose K, et al. Clinical utility of measuring epicardial adipose tissue thickness with echocardiography using a high-frequency linear probe in patients with coronary artery disease. J Am Soc Echocardiogr. 2015;28(1240-6):e1.

48. Jang HC, Lee HK, Lee H, Cha JG, Kim YS, Cho JH. Analyzing correlation between epicardial fat area and metabolic syndrome risk factor by using lowdose Lung CT. Pak J Med Sci. 2015;31:1207-12.

49. Homsi R, Meier-Schroers M, Gieseke J, et al. 3Ddixon MRI based volumetry of peri- and epicardial fat. Int J Cardiovasc Imaging. 2016;32:291-9.

50. McGavock JM, Lingvay I, Zib I, et al. Cardiac steatosis in diabetes mellitus: a $1 \mathrm{H}$-magnetic resonance spectroscopy study. Circulation. 2007; 116:1170-5.

51. Rijzewijk LJ, van der Meer RW, Smit JW, et al. Myocardial steatosis is an independent predictor of diastolic dysfunction in type 2 diabetes mellitus. J Am Coll Cardiol. 2008;52:1793-9.

52. Kankaanpaa M, Lehto HR, Parkka JP, et al. Myocardial triglyceride content and epicardial fat mass in human obesity: relationship to left ventricular function and serum free fatty acid levels. J Clin Endocrinol Metab. 2006;91:4689-95. 
53. Habibi J, Aroor AR, Sowers JR, et al. Sodium glucose transporter 2 (SGLT2) inhibition with empagliflozin improves cardiac diastolic function in a female rodent model of diabetes. Cardiovasc Diabetol. 2017;16:9.

54. Majewski C, Bakris GL. Blood pressure reduction: an added benefit of sodium-glucose cotransporter 2 inhibitors in patients with type 2 diabetes. Diabetes Care. 2015;38:429-30.

55. Shigiyama F, Kumashiro N, Miyagi M, et al. Effectiveness of dapagliflozin on vascular endothelial function and glycemic control in patients with early-stage type 2 diabetes mellitus: DEFENCE study. Cardiovascular Diabetology. 2017;16:84.

56. Tremblay AJ, Lamarche B, Deacon CF, Weisnagel SJ, Couture P. Effects of sitagliptin therapy on markers of low-grade inflammation and cell adhesion molecules in patients with type 2 diabetes. Metabolism. 2014;63:1141-8.

57. Satoh-Asahara N, Sasaki Y, Wada H, et al. A dipeptidyl peptidase- 4 inhibitor, sitagliptin, exerts antiinflammatory effects in type 2 diabetic patients. Metabolism. 2013;62:347-51.
58. Shigiyama F, Kumashiro N, Miyagi M, et al. Linagliptin improves endothelial function in patients with type 2 diabetes: a randomized study of linagliptin effectiveness on endothelial function. J Diabetes Investig. 2017;8:330-40.

59. Hibuse T, Maeda N, Kishida K, et al. A pilot threemonth sitagliptin treatment increases serum adiponectin level in Japanese patients with type 2 diabetes mellitus-a randomized controlled trial START-J study. Cardiovasc Diabetol. 2014;13:96.

60. Merovci A, Solis-Herrera C, Daniele G, et al. Dapagliflozin improves muscle insulin sensitivity but enhances endogenous glucose production. J Clin Invest. 2014;124:509-14.

61. Obata A, Kubota N, Kubota T, et al. Tofogliflozin improves insulin resistance in skeletal muscle and accelerates lipolysis in adipose tissue in male mice. Endocrinology. 2016;157:1029-42.

62. Peterson LR, Herrero P, Schechtman KB, et al. Effect of obesity and insulin resistance on myocardial substrate metabolism and efficiency in young women. Circulation. 2004;109:2191-6. 